\title{
Influência do exercício físico na sintomatologia de mulheres climatéricas
}

\author{
Influeneof physicd exeriseinthesymtonatogy of dimateric nomen
}

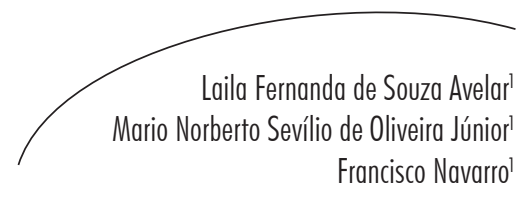

\section{Resumo}

O objetivo deste estudo foi verificar as alterações da sintomatologia climatérica em mulheres, decorrentes da prática de um programa de exercício físico aeróbio. Constituiu pesquisa descritiva do tipo experimental e cunho quantitativo. Participaram do estudo oito mulheres com faixa etária 55,25 $\pm 2,71$ anos, estatura 1,49 $\pm 0,04 \mathrm{~m}$, massa corporal $64,55 \pm 14,73 \mathrm{Kg}$, IMC $29,98 \pm 5,48 \mathrm{Kg} / \mathrm{m}^{2}$, e pressão arterial média $120 / 82 \mathrm{mmHg}$, que executaram programa de exercícios físicos (oito semanas), em dois estágios de quatro semanas cada, sendo em quatro delas sem controle da intensidade e em quatro com controle ( $70 \%$ FC) As participantes, ao iniciarem o protocolo tiveram avaliada sintomatologia a cada quatro semanas de exercício. Para a análise estatística dos dados, utilizaram-se a relação de média, desvio-padrão, valores máximos e mínimos, coeficiente de variância e o teste t para observações pareadas. Com efeito significativo em $5 \%$, os resultados obtidos foram a melhora na sintomatologia climatérica, segundo os escores do Índice Menopausal de Blatt Kupperman, com a prática do protocolo de exercício físico aeróbio proposto em $15,62 \%$ após quatro semanas, e 16,28\% após oito semanas; e melhora na capacidade aeróbia da classificação boa para excelente segundo a American Heart Association, com ganhos de 27,82\%. Conclui-se que a execução do protocolo de exercício físico proposto trouxe melhoras na sintomatologia climatérica nas mulheres participantes do estudo nos dois estágios, com melhora total de $23,85 \%$, apesar da duração de apenas oito semanas.

\section{Abstract}

This study aimed to determine the changes associated with the symptoms in menopausal women with the practice of an aerobic exercise program. It is a descriptive research of experimental and quantitative type. Eight women participated, in the age group 55.25 \pm 2.71 years, height $1.49 \pm 0.04 \mathrm{~m}$, body mass $64.55 \pm 14.73 \mathrm{~kg}$, BMI $29.98 \pm 5.48 \mathrm{~kg} /$ $\mathrm{m}^{2}$, and mean blood pressure $120 / 82 \mathrm{mmHg}$, performing a physical exercise program

\footnotetext{
Laboratório de Fisiologia e Prescrição de Exercício, Departamento de Educação Física. Universidade Federal do Maranhão. São Luis, MA, Brasil.
}

\section{Palavras-chave :}

Envelhecimento. Mulheres. Climatério. Sintomas. Exercício Físico. Análise Estatística. 
(8 weeks) in two stages of four weeks each, but four of them without control of intensity and the other four with control $(70 \%$ HT). They had to start the protocol symptoms assessed every four weeks of exercise. For statistical analysis it was used the ratio of mean, standard deviation, maximum and minimum coefficient of variance and test for paired observations. With significant effect at $1 \%$, the results were improvement in climacteric symptoms according to the scores of Blatt Kupperman Menopausal Index, with the practice of aerobic physical exercise protocol proposed in $15.62 \%$ after four weeks, and 16.28\% after eight weeks; and improvement of aerobic capacity from good to excellent classification according to the second American Heart Association, with gains of $27.82 \%$. It is concluded that implementing the proposed protocol exercise brought improvement in climacteric symptoms in women participating in the study in both stages, with a total improvement of $23.85 \%$, although in only eight weeks.
Key words: Aging. Women. Climacteric. Symptoms. Exercise. Statistical Analysis.

\section{INTRODUÇÃO}

O termo "climatério", derivado do grego Klimater (ponto crítico), envolve a meia idade que vai desde os 40 aos 60 anos e é considerado uma fase natural da vida da mulher., ${ }^{1,2}$ Constitui a transição entre a vida reprodutiva e nãoreprodutiva da mulher, compreendendo um longo período que começa pela passagem do ciclo ovulatório potencialmente fértil para um período de falência ovariana. ${ }^{3-5}$

As diversas alterações fisiológicas típicas desta fase podem resultar em queixas, das quais as que afetam a maioria das mulheres são: ondas de calor, sudorese, calafrios, palpitações, cefaleia, tonturas, parestesia, insônia, perda de memória e fadiga. ${ }^{6,7}$ As ondas de calor podem vir acompanhadas de rubor, sudorese, calafrios, palpitações ou episódios de taquicardia. Consistem em sensação de calor que se irradia da porção superior do tórax para o pescoço e cabeça, acompanhando-se de sudorese profunda. São mais desagradáveis à noite, determinando agitação, insônia e fadiga; durante os episódios há elevação da temperatura cutânea. ${ }^{6}$

Desta forma, mulheres sintomáticas tendem a ter desconfortos que interferem no relacionamento familiar, adaptação sexual e integração social. ${ }^{8}$ A mulher se afasta do ambiente e se retrai, quando é o momento de ampliar o campo das relações - este comportamento prejudica ainda mais seu estado de saúde geral.
A prática de exercício físico para alívio da sintomatologia tem sido recomendada como tratamento alternativo, ${ }^{1,9}$ já que a inatividade física, mais prevalente entre as mulheres no climatério, pode favorecer o surgimento de sintomas e das posteriores patologias. Essa prevalência foi evidenciada em estudo feito no Ambulatório de Climatério de Caxias do Sul (UCS), no qual, das 323 mulheres pesquisadas, pode-se observar que $78,6 \%$ da amostra não praticam atividades físicas regulares. ${ }^{10}$ Em estudos de Murata \& Schimer ${ }^{11}$ e Piazza, De Lorenzi \& Sacilato ${ }^{12}$ também foi destacado o significativo percentual de inatividade física das mulheres neste período. O exercício físico pode ser definido como uma subcategoria de atividade física planejada, estruturada e repetitiva, com o objetivo de ganhar e manter a performance física ${ }^{13} \mathrm{e}$, dentre outras finalidades, para promoção e manutenção da saúde.

Assim, para a mulher em climatério, existe uma relação inversa entre o exercício praticado regularmente e as principais causas de morte nestes indivíduos. ${ }^{14}$ Diversos estudos têm demonstrado benefícios do exercício no controle e prevenção primária e secundária de coronariopatia, obesidade, hipertensão arterial, osteoporose e diabetes mellitus prevalentes nesse período de declínio hormonal (hipoestrogenismo), ${ }^{1}$ bem como para os sintomas citados anteriormente, com destaque para os efeitos salutares do exercício sobre os fogachos e a depressão psíquica no climatério.' Dessa forma, são recomendadas atividades de variadas naturezas, dando-se prioridade aos exercícios aeróbicos. ${ }^{1}$ 
Com efeito, de posse do conhecimento existente sobre a importância do exercício físico para o público em questão, este estudo tem por objetivo verificar as alterações da sintomatologia climatérica em mulheres, decorrentes da prática de um programa de exercício físico aeróbio.

\section{MATERIAIS E MÉTODOS}

\section{Participantes}

Oito mulheres climatéricas foram recrutadas por meio de anúncio afixado no mural do Núcleo de Esportes da Universidade Federal do Maranhão (UFMA). Para tanto, das 12 mulheres que compareceram, apenas oito foram selecionadas para este estudo, por estarem de acordo com os critérios de inclusão, que foram: mulheres com faixa etária entre 40 e 60 anos e relatarem não praticar exercício físico (consideradas sessões de 30 minutos, três vezes na semana) há pelo menos seis meses antes da coleta de dados; estar apta a realizar exercícios físicos; apresentar disponibilidade para participar das sessões de treinos no Laboratório de Reabilitação Psicomotriz e Orgânica (LAREPO) localizado na UFMA; durante a pesquisa não ingressar em outros programas de exercícios físicos e não faltar mais que três vezes ao treinamento durante o período do estudo. Todas foram informadas sobre os procedimentos e suas implicações, e confirmaram sua participação assinando o termo de consentimento livre e esclarecido. A pesquisa foi aprovada pelo Comitê de Ética em Pesquisa da instituição onde o experimento foi realizado (processo CEP-UFMA 23115-003465/2011-88).

\section{Delineamento experimental}

A pesquisa caracterizou-se como sendo descritiva do tipo experimental e de cunho quantitativo. Inicialmente, foi realizada avaliação física para aferição das seguintes variáveis: estatura, massa corporal, índice de massa corporal (IMC), \% gordura corporal (\%GC), frequência cardíaca/repouso (FCR), pressão arterial sistólica/repouso (PASR), pressão arterial diastólica/repouso (PADR). No mesmo dia, foi aplicado o Índice Menopausal de Blatt e Kupperman (IMBK), ${ }^{15}$ para determinar sintomatologia climatérica.

O protocolo de exercício consistia em 50 minutos de caminhada em esteira rolante da Moviment Technology, modelo RT150PRO. As sessões de treinamento eram realizadas três vezes por semana, sob monitoria dos acadêmicos de Educação Física e supervisão do coordenador da pesquisa.

O referido protocolo teve duração de oito semanas, dividido em dois estágios de quatro semanas cada. $O$ primeiro correspondeu às quatro semanas iniciais para adaptações fisiológicas das participantes que eram inativas fisicamente, em que a intensidade foi controlada apenas pela percepção de esforço das participantes; ao final, foi aplicado o IMBK individualmente, por meio do preenchimento do índice por um único avaliador, com base nas respostas das participantes.

A partir do início da quinta semana, foram avaliados o $\mathrm{VO}_{2}$ máx. e a frequência cardíaca máxima (FC. máx.) por meio de teste de esforço utilizando o protocolo de Bruce, Kusume \& Hosmer. ${ }^{16}$ Procedeu-se às sessões de treino, acrescentando intensidade na caminhada (70\% FC. máx.), que constituiu o segundo estágio. A frequência cardíaca (FC) foi monitorada por meio de monitor de frequência cardíaca da marca Polar modelo FT1. Ao final da oitava semana, novamente foram aplicados o IMBK e o teste de $\mathrm{VO}_{2}$ máximo.

\section{Avaliação física}

Para a avaliação da estatura, utilizou-se um estadiômetro acoplado a uma balança. Desta forma, a participante posicionava-se com os calcanhares, glúteos, dorso e cabeça encostados no plano vertical da escala. ${ }^{17}$ Para a determinação da massa corporal, foi utilizada uma balança mecânica, marca Filizola, com precisão de $100 \mathrm{~g}$, assim os dados foram obtidos estando as avaliadas com um mínimo de roupa possível, seguindo padronização. ${ }^{18} \mathrm{O}$ índice de 
massa corporal (IMC) foi calculado com base nas medidas de massa corporal $(\mathrm{kg})$ e estatura (m), aplicando a fórmula: IMC $=$ massa/altura ${ }^{2}$. $\mathrm{E}$, por meio da equação [\%Gordura $=1,20$ $(\mathrm{IMC})+$ 0,23 (Idade) - 10,8 (Sexo) - 5,4], foi realizado o cálculo para estimativa do \%gordura corporal $(\% \mathrm{GC}){ }^{19}$

O monitor cardíaco foi utilizado em todos os testes, seguindo seus procedimentos para controle da FC. Na mensuração da pressão arterial de repouso, a técnica de medida empregada foi o método auscultatório na artéria braquial, com base nos sons de Korotkoff. Para que fosse possível identificar e registrar esses sons, tornou-se necessário dispor de campânula de estetoscópio e esfigmomanômetro, ambos da marca G-Tech da linha Premiun. O indivíduo permanecia em lugar calmo durante cinco minutos, e em seguida se realizava a aferição.

\section{Índice Menopausal de Blatt e Kupperman (IMBK)}

Índice referência para avaliar os sintomas climatéricos das participantes, consiste na avaliação de vários sintomas, medidos de acordo com a intensidade de acometimentos referidos pelo grupo pesquisado. Avalia os seguintes sintomas ou queixas: sintomas vasomotores, insônia, parestesia, nervosismo, melancolia, vertigem, fraqueza, artralgia/mialgia, cefaleia, palpitação e formigamento.

A cada uma dessas queixas são atribuídas diferentes pontuações, segundo sua intensidade e prevalência. Os escores totais são classificados em leves (valores até 19), moderados (entre 20 e
35) ou intensos (maior que 35). Assim, quanto maior a pontuação obtida, mais intensa a sintomatologia climatérica. ${ }^{15}$

\section{Avaliação da capacidade física}

O teste de Bruce, Kusumi \& Hosmer ${ }^{16}$ é um protocolo para estimativa de $\mathrm{VO}_{2}$ máximo por meio de ergômetro, a esteira rolante. O públicoalvo são sedentários e ativos de ambos os gêneros, cardíacos e idosos. Possui três equações para predição, a utilizada foi $\mathrm{VO}_{2}$ máx. $\mathrm{ml}^{-1} \cdot \mathrm{kg}^{-1}$. $\min ^{-1}=4,38 .(\mathrm{T})-3,90$.

\section{Análise estatística}

Para a análise estatística dos resultados, utilizou-se a relação de média e desvio-padrão para os valores no primeiro e segundo estágio, e foi aplicado, ainda, o teste $t$ de Student para verificação das diferenças entre os valores de $\mathrm{VO}_{2}$ máx. e sintomatologia (IMBK) antes e após o protocolo de exercício, no valor de significância para rejeição da hipótese de nulidade $\mathrm{p} \leq 0,05$. Os resultados foram apresentados em forma de tabelas.

\section{RESULTADOS}

Com o objetivo de visualizar as características descritivas dos indivíduos do estudo, a tabela 1 apresenta os escores médios de idade, massa corporal, estatura, IMC, \% GC, PAS/rep., PAD. rep., FC rep., com seus respectivos desvio-padrão (dp) e amplitude. 
Tabela 1 - Características físicas e morfológicas das mulheres climatéricas de 52 a 58 anos de idade. São Luis, MA, 2011.

\begin{tabular}{lccc}
\hline \multicolumn{1}{c}{ Variáveis $(\mathrm{n}=8)$} & Média $\pm \mathrm{dp}$ & Mínimo & Máximo \\
\hline Idade(anos) & $55,25 \pm 2,71$ & 52 & 58 \\
Massa corporal(Kg) & $64,55 \pm 14,73$ & 47 & 95 \\
Estatura(M) & $1,49 \pm 0,04$ & 1,41 & 1,55 \\
$\mathrm{IMC}\left(\mathrm{Kg} / \mathrm{m}^{2}\right)$ & $29,98 \pm 5,48$ & 22,21 & 39,54 \\
Gordura Corporal $(\%)$ & $43,15 \pm 6,49$ & 33,44 & 54,23 \\
PAS repouso (mmHg) & $120 \pm 12,88$ & 100 & 138 \\
PAD repouso $(\mathrm{mmHg})$ & $82 \pm 17,23$ & 60 & 110 \\
FC repouso $(\mathrm{bpm})$ & $71,3 \pm 7,21$ & 60 & 80 \\
\hline
\end{tabular}

Com base na avaliação física realizada antes do início das sessões de treinamento, verificouse um grupo cujos indivíduos possuem faixa etária média de 55 anos; média para IMC de $29,98 \mathrm{Kg} / \mathrm{m}^{2} ; \%$ GC com média 43,15; e pressão arterial média de 120/82 $\mathrm{mmHg}$ (tabela 1).
Em relação à sintomatologia do grupo em estudo, pode-se observar que houve melhora progressiva quando se comparam os valores (tabela 2) do IMBK obtidos antes e após o primeiro e segundo estágio de treinamento, sem e com controle da intensidade pela frequência cardíaca e do $\mathrm{VO}_{2}$ máximo, respectivamente.

Tabela 2 - Dados das alterações da sintomatologia climatérica durante o treinamento físico. São Luis, MA, 2011.

\begin{tabular}{lccc}
\hline \multicolumn{1}{r}{ Período $(\mathrm{n}=8)$} & Média $\pm \mathrm{dp}$ & Mínimo & Máximo \\
\hline INÍCIO & $24,62 \pm 4,71$ & 18 & 31 \\
PRIMEIRO ESTÁGIO & $21,62 \pm 4,40$ & 16 & 28 \\
SEGUNDO ESTÁGIO & $18,75 \pm 3,65$ & 14 & 24 \\
\hline
\end{tabular}

* Diferença entre o $1^{\circ}$ e $2^{\circ}$ estágio de $\mathrm{p}<0,001$ 
A capacidade física dos sujeitos no segundo estágio foi avaliada por testes. A tabela 3 apresenta escores médios e desvio-padrão e amplitudes dos valores coletados. Ressalta-se que os valores foram aumentando à medida que eram feitas as avaliações, evidenciando-se progressos com o treinamento.

Tabela 3 - Dados da avaliação do $\mathrm{VO}_{2}$ máximo $\left(\mathrm{ml}^{-1} \cdot \mathrm{kg}^{-1} \cdot \mathrm{min}^{-1}\right)$.

\begin{tabular}{cccc}
\hline Período $(\mathrm{n}=8)$ & Média $\pm \mathrm{dp}$ & Mínimo & Máximo \\
\hline Início do $2^{\circ}$ estágio & $31,64 \pm 7,51$ & 20,35 & 42,54 \\
Final do $2^{\circ}$ estágio & $43,83 \pm 9,55$ & 28,68 & 55,01 \\
\hline
\end{tabular}

* Diferença entre o $1^{\circ}$ e $2^{\circ}$ estágio correspondendo $\mathrm{p}=0,000653952$

No presente estudo, encontramos uma diferença estatisticamente significativa nas duas variáveis pesquisadas de $\mathrm{p} \leq 0,01$. Assim, quando analisados os valores diferença IMBK entre o início e $1^{\circ}$ estágio, tem-se 0,000633325; diferença IMBK entre o $1^{\circ}$ e o $2^{\circ}$ estágio, obteve-se $\mathrm{p}=0$, 000544452; para diferença $\mathrm{VO}_{2}$ máx. entre o $1^{\circ}$ estágio e $2^{\circ}$ estágio, $\mathrm{p}=0,000653952$.

\section{DISCUSSÃO}

As mulheres voluntárias deste estudo possuíam média etária 55,25 $\pm 2,71$ anos e se enquadram na fase do climatério chamada perimenopausa (45 aos 55 anos), considerada mais propensa a sintomatologia e acometimento de doenças. ${ }^{20,21} \mathrm{O}$ IMC indicou sobrepeso ${ }^{16}$ motivo de atenção (tabela 1), já que, segundo estudos, o IMC alcança o pico máximo entre 50 e 59 anos, devido à redução da lípase lipoproteica e estrogênio, responsável por regular o acúmulo de gordura e sua distribuição nos tecidos; lentidão metabólica e redução da atividade física; risco elevado de doenças cardiovasculares, endócrinas e neoplasias, ${ }^{1}$ o que confirma o excesso de gordura corporal.

Observamos, ainda, que o valor médio do $\%$ GC de 43,15 $\pm 6,49$ estava fora do ideal de $23 \%$ para idade; no entanto, a substituição gradativa da musculatura por tecido adiposo faz parte do processo de envelhecimento. ${ }^{22}$
Quanto à pressão arterial, os valores médios de 120/82 mmHg para PAS/PAD mensurados em repouso são consideradas dentro do padrão de controle. ${ }^{1}$ É preciso destacar que obesidade e hipertensão arterial são fatores de risco para doenças cardiovasculares mais frequentes com hipoestrogenismo no período do climatério, associados ao processo de envelhecimento.

A sintomatologia climatérica é referenciada por aproximadamente $70 \%$ das mulheres, seja nos âmbitos físico, emocional ou social, em que as instabilidades hormonais são fatores principais desse processo. $^{23}$ No presente estudo, com base na classificação de Kupperman \& Blatt, ${ }^{15}$ obtivemos nível moderado para a sintomatologia dos indivíduos antes da prática de exercício. Após o primeiro estágio de treino de intensidade, este continuou moderado e após o segundo estágio, alterou-se para nível leve.

É possível, assim, realizar a seguinte análise: em relação aos escores dos sintomas, houve redução de três nos dois estágios. Porém, ao se comparar o valor do início até o final da oitava semana, percebe-se um ganho maior, visto que os mesmos permutaram de um nível para outro com decréscimo de aproximadamente seis escores. Em percentuais, houve redução do início ao final do $1^{\circ}$ estágio, de $15,62 \%$; deste para o final do $2^{\circ}$ estágio, de $16,28 \%$; e do início ao final do $2^{\circ}$, de $23,85 \%$. 
Tairova\& De Lorenzi ${ }^{24}$ relataram que, enquanto 63,6\% das mulheres sedentárias descreveram sintomas climatéricos de intensidade moderada a severa, o mesmo foi referido por 33,4\% do grupo fisicamente ativo. Além disso, as mulheres desse grupo não relataram sintomas muito severos, ao contrário de 3,8\% das sedentárias.

Emestudotipocaso-controle,${ }^{25}$ quando ogrupo submetido a exercícios foi comparado ao que não praticava atividades físicas regulares, identificouse também um decréscimo da severidade de sintomas menopausais no período climatérico (58,3\% contra $66,7 \%$, respectivamente). Já em pesquisa $^{26}$ envolvendo 133 mulheres com idade de 44 a 60 anos, submetidas a atividades físicas durante 12 meses, estudiosos concluíram que, além de melhora da percepção de severidade dos sintomas menopausais, o sentimento de vigor físico atua positivamente na sensação psicológica de bem-estar, na vitalidade, na saúde mental e nos aspectos emocionais, embora essa relação possa ser mediada por outros fatores.

O exercício físico é recomendado tradicionalmente para mulheres, pois atua na promoção, prevenção e manutenção da saúde. Em posicionamento oficial sobre Atividade Física e Saúde na Mulher, ${ }^{14}$ um programa de exercício físico ideal deve ser realizado na maior parte dos dias da semana, com duração das sessões variando entre 30 e 90 minutos, intensidade moderada, como 40 a 75\% do VO2 máx. ou 55 a 85\% da FC máxima.

Seguindo tal recomendação, nosso protocolo foi planejado e, por meio do teste de capacidade física, podem-se comprovar as alterações decorrentes da prática do exercício, ou seja, melhora nos escores. Assim, no início do segundo estágio, o valor era $31,64 \pm 7,51$; ao final

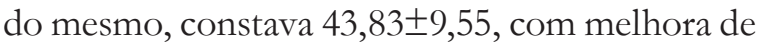
$27,81 \%$. Este resultado coincide com os relatados por outros autores, segundo os quais ocorreria aumento de 10 a $25 \%$ no consumo máximo de oxigênio após treinamento aeróbico. ${ }^{27-29}$

De acordo com a classificação da American Heart Association, ${ }^{30}$ com esses valores a classificação seria boa e excelente, respectivamente. Desta forma, o presente estudo também verificou melhora significativa no $\mathrm{VO}_{2}$ máx. das participantes, apontando que a intensidade e o modelo de exercício foram benéficos para a melhoria da aptidão física das mulheres submetidas ao programa.

Em estudo caso-controle, ${ }^{24}$ revelou-se que a capacidade cardiorrespiratória segundo a estimativa do consumo máximo de oxigênio $\left(\mathrm{VO}_{2}\right.$ máx.) também era significativamente maior entre as mulheres fisicamente ativas $(\mathrm{p}<0,01)$, atingindo valores médios ao redor de $26,5 \pm 7,0 \mathrm{ml} / \mathrm{Kg} / \mathrm{min}$, enquanto que, entre as sedentárias, atingiu valores médios ao redor de 20,2 $\pm 7,8 \mathrm{ml} / \mathrm{Kg} / \mathrm{min}$. Assim, as mulheres praticantes de atividade física, ainda que de intensidade leve a moderada, mostraram capacidade funcional (níveis de $\mathrm{VO}_{2}$ máx.) significativamente maior do que as mulheres sedentárias, considerando que a média etária entre os dois grupos era estatisticamente semelhante.

Algumas limitações metodológicas deste estudo merecem considerações: o tamanho amostral pode comprometer algumas possíveis associações estatísticas (a não-utilização de um grupo controle), mas ainda assim fornece informações importantes a respeito do tema. Ainda que seus achados não possam ser extrapolados para a população em geral, espera-se que estes estimulem novas pesquisas voltadas a aprofundar o conhecimento sobre a interferência da prática de exercício físico na sintomatologia climatérica.

\section{CONCLUSÃO}

Com base na execução do protocolo de exercício físico proposto, realizado três vezes por semana durante oito semanas, verificamos melhoras significativas na sintomatologia nos dois estágios $-15,62 \%$ e $16,28 \%$, para o $1^{\circ}$ e $2^{\circ}$, respectivamente; bem como aumento do $\mathrm{VO}_{2}$ máx. (melhora de 27,81\%) da presente amostra.

Tais resultados ajudam a reforçar a importância da realização de exercício físico aeróbio para mulheres em climatério, período de transformação reprodutiva em que enúmeras queixas são frequentes. É necessário, no entanto, 
que sejam feitas mais investigações, inclusive com amostra maior e maior tempo de treinamento, e ainda com a utilização de grupo-controle. Nesta perspectiva, será possível produzir conhecimento importante que contribua positivamente para o estado de saúde de várias mulheres.

\section{REFERÊNCIAS}

1. Brasil. Ministério da Saúde. Secretaria de Políticas Públicas. Área Técnica da Saúde da Mulher. Manual de atenção à mulher no climatério e menopausa. Brasília: Ministério da Saúde, 2008.191p.

2. Consenso Brasileiro Multidisciplinar de Assistência à Saúde da Mulher Climatérica. São Paulo: SOBRAC; Rio de Janeiro: FEBRASGO; 2003. 254p.

3. De Lorenzi D.R.S, Danelon C, Saciloto B, Padilha Jr I. Fatores Indicadores da sintomatologia Climatérica. Rev Bras Ginecol Obst .2005. 27 (1): 7-11.

4. Renó Júnior, J. Estudo Randomizado Duplo Cego de Avaliação dos Efeitos de Terapia de Reposição Estrogênica sobre Funções Cognitivas e Psicomotoras em Mulheres Menopausadas [tese de Doutorado]. São Paulo: Faculdade de Medicina da Universidade de São Paulo - Departamento de Psiquiatria; 2002.

5. Secco, LM. Mulher e sexualidade: estudo realizado a partir das deusas mitológicas. Rev. Terapia Sexual. Clin. - Pesquisa e aspectos Psicossociais. 2002; 5(1): 41-62.

6. Freitas KM, Silva ARV, Silva RM. Mulheres vivenciando o climatério. Acta Scientiarum Health Sciences 2004; 26(1): 121-28.

7. Santos, L M, Eserian PV, Rachid LP, Cacciatore A, Bourget IMM, Rojas AC. Síndrome do climatério e qualidade de vida: uma percepção das Mulheres nessa fase da vida. Revista APS 2007 jan / jun.10(1): $20-6$.

8. Lopes, GP. Sexualidade no Climatério. In: Rodrigues EJS, Filho JD, organizadores. Menopausa: seja bemvinda e bem vivida. Rio de Janeiro (RJ): Medis;2001. p.43-53.

9. Jales, W. Os Benefícios dos Exercícios Físicos No Climatério e Menopausa. Motriz. 2009.15(2) (Supl.1), p.S1-S456.

10. De Lorenzi DRS, Baracat EC, Saciloto B, Padilha Jr I. Fatores associados à qualidade de vida pós menopausa. Rev Assoc Med Bras. 2006; 52(5): 312-7.

11. Murata. I M H; Schirmer. J. Manifestações biopsicogênicas decorrentes do climatério entre trabalhadoras de uma universidade pública. Acta Paul. Enf. 2004,17(2):164-171.
12. Piazza IP, De Lorenzi DRS, Sacilato B. Avaliação do risco cardiovascular entre mulheres climatéricas atendidas em um programa de saúde da família. Rev Gaúcha Enferm. 2005; 26(2):200-9.

13. González FJ, Fensterseifer, PE. (org.). Dicionário Crítico de Educação Física. Ijuí: Ed. Unijuí, 2005.421p.

14. Leitão MB, Lazzoli Jk, De Oliveira MAB, Da Nóbrega ACL, Silveira GG, De Carvalho T. Posicionamento Oficial da Sociedade Brasileira de Medicina do Esporte: Atividade Física e Saúde na Mulher. Rev Bras Med Esporte. 2000 nov / dez; 6 (6): 215-220.

15. Kupperman HS.; Blatt MHG. Menopausal indice. J Clin Endocrinol.1953; 13(1):688-694.

16. Kenneth F Hossack MD.; Robert A.Bruce, MD.; FACC.; Bert GREEN MD.; Fusako Kusumi MS.; Timothy A. De ROUEN.; Trimble S. Maximal oxygen intake and nomographic assessment of functional aerobic impairment in cardiovascular disease. American Heart Journal. 1973;85(4).546-62.

17. Berquó, E.; Marques, R.M. Crescimento e desenvolvimento pubertário em crianças e adolescentes brasileiros: II - Altura e Peso. São Paulo(SP): Editora Brasileira de Ciências, 1982.364p.

18. De Rose EH. Cineantropometria, educação física e treinamento desportivo. Rio de Janeiro : FAE, Brasília : SEED, 1984.412p.

19. WHO (World Health Organization). Obesity: Preventing and Managing the Global Epidemic. Report of a WHO Consultation on Obesity. Geneva: WHO. 1998.

20. Bossemeyer R. Aspectos gerais do climatério. In. Fernandes C. E (Org.) Climatério Feminino. São Paulo; Lemos. 1999. p.17-33.

21. Blumel M, et al. Prevalência de sintomas psíquicos y vasomotores en diferentes periodos del climaterio. Rev.Chil. Obstt. Ginecol, Santiago. 1997; 62(6): 412-8.

22. CR, Petroski EL, Da Silva RCR, Da Silva JCN. Indicadores antropométricos de excesso de gordura corporal em mulheres*. Rev Bras Med Esporte. 2006 mai / jun ;12 (3): 119-124. 
23. Daoud IG. Encontros e desencontros das mulheres climatéricas que buscam assistência em serviço do sistema único de saúde. R. Enfermagem Rio de Janeiro. 2002;10(1): 33-7.

24. Tairova OS, De Lorenzi DRS. Influência do exercício físico na qualidade de vida de mulheres na pós-menopausa: um estudo caso-controle. Rev. Bras. Geriatr. Gerontol. 2011; 14(1):135-145.

25. Villaverde-Gutiérrez C, Araújo E, Cruz F, Roa JM, Barbosa W, Ruíz.Villaverde G. Quality of life of rural menopausal women in response to a customized exercise programme. J Adv Nurs. 2006;54(1):11-9.

26. S, McAuley E. Physical activity, symptoms, esteem, and life satisfaction during menopause. Maturitas. 2005;52 (3-4):374-385.
27. Spina RJ, Ogawa T, Kohrt WM. Differences in cardiovascular adaptations to endurance exercise training between older men and women. J Appl Physiol 1993;75(2):849-55.

28. Macedo IF, Duarte CR, Matsudo VKR. Análise da potência aeróbica em adultos de diferentes idades. Rev. Bras. Ciên. Mov. 1987; 1(1):7-13.

29. Okada, GT.; Silva, RG.Efeito da freqüência semanal de treinamento sobre variáveis físicas e hemodinâmicas de mulheres entre 30 e 50 anos de idade. Motriz. 2005;11(1) (Supl.), p. S7-S200

30. American Heart Association (1972). Exercise Testing of Apparently Individuals: A Handbook for Physicians. Dallas, TX: American Heart Association. 